ICRR-Report-405-97-28

OCHA-PP-108

\title{
$C P$ violation in the top-quark system through light squarks
}

\author{
Mayumi Aoki ${ }^{1}$ 周 and Noriyuki Oshimo ${ }^{2}$ \\ ${ }^{1}$ Graduate School of Humanities and Sciences, Ochanomizu University \\ Otsuka 2-1-1, Bunkyo-ku, Tokyo 112, Japan \\ 2 Institute for Cosmic Ray Research, University of Tokyo \\ Midori-cho 3-2-1, Tanashi, Tokyo 188, Japan
}

\begin{abstract}
We study a decay rate asymmetry of the top quark in the supersymmetric standard model, taking into account the constraints from the electric dipole moments of the neutron and the electron. One $C P$-violating phase contained in the masssquared matrices of squarks and sleptons is allowed to have an unsuppressed value, even if their masses are of order $100 \mathrm{GeV}$. Sizable $C P$ violation could then occur in the production and decay of the top quark through interactions with top squarks. The asymmetry between the widths for the decays $t \rightarrow b W^{+}$and $\bar{t} \rightarrow \bar{b} W^{-}$can be of order $10^{-3}$, which will possibly be detectable in the near future.
\end{abstract}

PACS: 11.30.Er, 12.60.Jv, 13.40.Em, 14.65.Ha

Keywords: $C P$ violation; Supersymmetry; Top quark; EDMs

\footnotetext{
${ }^{*}$ Research Fellow of the Japan Society for the Promotion of Science.
} 


\section{Introduction}

Up to now $C P$ violation has only been found in the $K^{0}-\bar{K}^{0}$ system, which is well described by the Kobayashi-Maskawa (KM) mechanism of the standard model (SM) [回]. In near-future experiments at $B$ factories, $C P$-violating phenomena are expected to be observed in the $B^{0}-\bar{B}^{0}$ system, possibly providing confirmation of the KM mechanism. On the other hand, baryon asymmetry of our universe may be an outcome of $C P$ violation, though cannot be generated through the KM mechanism. Furthermore, many extensions of the SM predict new sources of $C P$ violation. Therefore, various possibilities of examining $C P$ violation should be studied theoretically and experimentally.

One of possible reactions for studying $C P$ violation is the production and decay of the top quark [2, 3, 4, 5, 6]. Although sizable $C P$ violation is not predicted within the framework of the SM, it could be induced by physics beyond the SM,

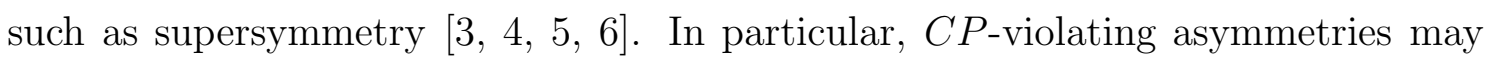
be observed in the angular or energy distributions of the particles arising from the top-quark decays. These asymmetries have been studied extensively.

In this paper we discuss another possible manifestation of $C P$ violation in the top-quark decay: an asymmetry between the partial decay rates for the decays $t \rightarrow b W^{+}$and $\bar{t} \rightarrow \bar{b} W^{-}$

$$
A_{C P}=\frac{\Gamma\left(t \rightarrow b W^{+}\right)-\Gamma\left(\bar{t} \rightarrow \bar{b} W^{-}\right)}{\Gamma\left(t \rightarrow b W^{+}\right)+\Gamma\left(\bar{t} \rightarrow \bar{b} W^{-}\right)} .
$$

We assume the supersymmetric standard model (SSM) based on $N=1$ supergravity coupled to grand unified theories (GUTs) [7], which could contain new $C P$-violating phases. For this asymmetry to be nonvanishing, in addition to $C P$ violation, it is necessary that the top quark has a decay mode which can yield a bottom quark and a $W$ boson through a final state interaction. We show that these two requirements can really be accommodated in the SSM, in spite of severe constraints imposed from the electric dipole moments (EDMs) of the neutron and the electron. The asymmetry could have a value of order $10^{-3}$, which will be detectable in the near future at e.g. LHC.

In the SSM, $C P$ violation in the production and decay of the top quark could be induced through interactions with the squarks of the third generation [3, 4, 5, 6]. The $C P$-violating effects of these interactions become sizable if relevant complex phases are not suppressed and the squark masses are of order $100 \mathrm{GeV}$. However, large $C P$-violating phases and small squark masses can easily lead to large magnitudes 
of the EDMs of the neutron and the electron. Since experimental upper bounds on these EDMs are fairly small, the possible $C P$-violating effects in the top-quark system have to be confronted with the constraints from the EDMs.

The neutron and electron EDMs strongly constrain $C P$ violation in the SSM. In general, their experimental bounds are satisfied if new $C P$-violating phases are much smaller than unity or squarks and sleptons are heavier than $1 \mathrm{TeV}$ [8]. In the ordinary scheme of the SSM, the masses of squarks and sleptons are not very different from each other, irrespective of the generations that they belong to. Also one of the new $C P$-violating phases is independent of the generations, and the other new phases are only slightly dependent on them. It is not likely that only the squarks of the third generation have masses of order $100 \mathrm{GeV}$ and $C P$-violating phases of order unity. Therefore, $C P$ violation in the top-quark system is severely constrained by the EDMs in the ordinary SSM. In the previous works for $C P$ violation in the top-quark system, it was assumed explicitly or implicitly that squark masses or $C P$-violating phases in the third generation are unrelated to those responsible for the EDMs of the neutron and the electron. The constraints from the EDMs were disregarded.

Reexamining the EDMs in the ordinary scheme of the SSM, we consider conditions of having squark masses of order $100 \mathrm{GeV}$ and an unsuppressed $C P$-violating phase. Under these conditions, one of the top squarks can be fairly light, while its interactions violate $C P$ invariance maximally. It is shown that in a certain parameter region the top quark can decay into a top squark $\tilde{t}$ and a neutralino $\chi$, being subsequently followed by a scattering which produces a bottom quark and a $W$ boson, as shown in Fig. 1. This decay process contributes to the $C P$ asymmetry $A_{C P}$ in Eq. (1).

This paper is organized as follows. In sect. 2 we summarize new sources of $C P$ violation in the SSM and present the interaction Lagrangians relevant to our discussions. In sect. 3 we discuss the SSM parameter values for which squarks are light and all the $C P$-violating phases are not suppressed, without giving too large values of the EDMs. In sect. 4 the decay rate asymmetry for the top quark is calculated. Analytic formulae of the asymmetry are given explicitly. Conclusions are given in sect. 5 . 


\section{$2 C P$-violating interactions}

The SSM contains new complex phases for $C P$ violation in addition to the KM phase in the Cabibbo-Kobayashi-Maskawa matrix. The parameters which have generally complex values are the $\mathrm{SU}(3), \mathrm{SU}(2)$, and $\mathrm{U}(1)$ gaugino masses $\tilde{m}_{3}, \tilde{m}_{2}$, and $\tilde{m}_{1}$, respectively, the Higgsino mass parameter $m_{H}$ in the bilinear term of the Higgs superfields, and the dimensionless coupling constants $A_{f}$ 's and $B$ in the trilinear and bilinear terms of the scalar fields, respectively. We assume unification for the gaugino masses at the GUT scale, giving the relation $\tilde{m}_{3} / \alpha_{3}=\tilde{m}_{2} / \alpha_{2}=3 \tilde{m}_{1} / 5 \alpha_{1}$. Also $A_{f}$ 's are assumed to have the same value of order unity at the GUT scale, so that their differences at the electroweak scale are small and thus can be neglected. Then, by redefining particle fields, we can take, without loss of generality, $m_{H}$ and $A_{f}$ as complex, and $\tilde{m}_{i}(i=1,2,3)$ and $B m_{H}$ as real. The complex phases of $m_{H}$ and $A_{f}$ are physical and become the origins of $C P$ violation, which we express as

$$
\begin{aligned}
m_{H} & =\left|m_{H}\right| \exp (i \theta) \\
A_{f} & =A=|A| \exp (i \alpha) .
\end{aligned}
$$

These conventions make the vacuum expectation values of the Higgs bosons $v_{1}$ and $v_{2}$ real.

The complex parameters lead to complex mass matrices for charginos $\omega$, neutralinos $\chi$, squarks $\tilde{q}$, and sleptons $\tilde{l}$. The mass matrices $M^{-}$and $M^{0}$ for the charginos and the neutralinos respectively are given by

$$
\begin{aligned}
M^{-}= & \left(\begin{array}{ccc}
\tilde{m}_{2} & -g v_{1} / \sqrt{2} \\
-g v_{2} / \sqrt{2} & m_{H}
\end{array}\right) \\
M^{0}= & \left(\begin{array}{cccc}
\tilde{m}_{1} & 0 & g^{\prime} v_{1} / 2 & -g^{\prime} v_{2} / 2 \\
0 & \tilde{m}_{2} & -g v_{1} / 2 & g v_{2} / 2 \\
g^{\prime} v_{1} / 2 & -g v_{1} / 2 & 0 & -m_{H} \\
-g^{\prime} v_{2} / 2 & g v_{2} / 2 & -m_{H} & 0
\end{array}\right) .
\end{aligned}
$$

These mass matrices are diagonalized to give mass eigenstates as

$$
\begin{aligned}
C_{R}^{\dagger} M^{-} C_{L} & =\operatorname{diag}\left(\tilde{m}_{\omega 1}, \tilde{m}_{\omega 2}\right) \quad\left(\tilde{m}_{\omega 1}<\tilde{m}_{\omega 2}\right) \\
N^{t} M^{0} N & =\operatorname{diag}\left(\tilde{m}_{\chi 1}, \tilde{m}_{\chi 2}, \tilde{m}_{\chi 3}, \tilde{m}_{\chi 4}\right) \quad\left(\tilde{m}_{\chi 1}<\tilde{m}_{\chi 2}<\tilde{m}_{\chi 3}<\tilde{m}_{\chi 4}\right)
\end{aligned}
$$

where $C_{R}, C_{L}$, and $N$ are unitary matrices. The mass of the gluinos $\tilde{g}$ is expressed by the $\mathrm{SU}(2)$ gaugino mass as $\tilde{m}_{3}=\left(\alpha_{3} / \alpha_{2}\right) \tilde{m}_{2}$.

The mass-squared matrix $M_{q}^{2}$ for the squarks corresponding to a quark $q$ with mass $m_{q}$, electric charge $Q_{q}$, and third component of the weak isospin $T_{3 q}$ is given 
by

$$
\begin{gathered}
M_{q}^{2}= \\
\left(\begin{array}{cc}
m_{q}^{2}+\cos 2 \beta\left(T_{3 q}-Q_{q} \sin ^{2} \theta_{W}\right) M_{Z}^{2}+\tilde{M}_{q L}^{2} & m_{q}\left(R_{q} m_{H}+A^{*} m_{3 / 2}\right) \\
m_{q}\left(R_{q} m_{H}^{*}+A m_{3 / 2}\right) & m_{q}^{2}+Q_{q} \cos 2 \beta \sin ^{2} \theta_{W} M_{Z}^{2}+\tilde{M}_{q R}^{2}
\end{array}\right), \\
R_{q}=\frac{1}{\tan \beta} \quad\left(T_{3 q}=\frac{1}{2}\right), \quad \tan \beta \quad\left(T_{3 q}=-\frac{1}{2}\right), \\
\tan \beta=\frac{v_{2}}{v_{1}},
\end{gathered}
$$

where $\tilde{M}_{q L}^{2}$ and $\tilde{M}_{q R}^{2}$ denote the mass-squared parameters for the left-handed squark and the right-handed squark, respectively, and $m_{3 / 2}$ is the gravitino mass. We have neglected generation mixings. The mass eigenstates $\tilde{q}_{1}$ and $\tilde{q}_{2}$ are obtained by diagonalizing the mass-squared matrix as

$$
S_{q}^{\dagger} \tilde{M}_{q}^{2} S_{q}=\operatorname{diag}\left(\tilde{M}_{q 1}^{2}, \tilde{M}_{q 2}^{2}\right) \quad\left(\tilde{M}_{q 1}^{2}<\tilde{M}_{q 2}^{2}\right)
$$

where $S_{q}$ is a unitary matrix. The slepton mass-squared matrices are obtained by appropriately changing $M_{q}^{2}$ in Eq. (7).

The masses of squarks and sleptons are related to each other. At the GUT scale, the mass-squared parameters for all the squarks and sleptons are considered to have a common value $m_{3 / 2}^{2}$ in supersymmetry-breaking terms. Then, at the electroweak scale, the values of the mass-squared parameters for the squarks of the first two generations and all the sleptons are approximately the same,

$$
\tilde{M}_{q L}^{2} \simeq \tilde{M}_{q R}^{2} \simeq \tilde{M}_{l L}^{2} \simeq \tilde{M}_{l R}^{2} \equiv \tilde{M}^{2}
$$

Those for the squarks of the third generation are expressed as

$$
\begin{array}{ll}
\tilde{M}_{t L}^{2}=\tilde{M}^{2}-c m_{t}^{2}, & \tilde{M}_{t R}^{2}=\tilde{M}^{2}-2 c m_{t}^{2} \\
\tilde{M}_{b L}^{2}=\tilde{M}^{2}-c m_{t}^{2}, & \tilde{M}_{b R}^{2}=\tilde{M}^{2}
\end{array}
$$

The parameters $\tilde{M}_{t L}^{2}, \tilde{M}_{t R}^{2}$, and $\tilde{M}_{b L}^{2}$ receive quantum corrections through Yukawa interactions proportional to the top-quark mass $m_{t}$, with $c=0.1-1$. Under this SSM scheme, all the squark and slepton masses are roughly the same, if $\tilde{M}$ is sufficiently larger than $m_{t}$. For $\tilde{M} \sim m_{t}$, the squark masses of the third generation become different from the other squark and slepton masses.

The complex mass matrices for the $R$-odd particles lead to $C P$-violating interactions. The interaction Lagrangians for these particles relevant to our study are given in the followings. 
The chargino-neutralino-W boson interactions:

$$
\begin{aligned}
& \mathcal{L}= \frac{g}{\sqrt{2}} W_{\mu}^{\dagger} \bar{\chi}_{j} \gamma^{\mu}\left(H_{L j i} \frac{1-\gamma_{5}}{2}+H_{R j i} \frac{1+\gamma_{5}}{2}\right) \omega_{i}+\text { H.c. }, \\
& H_{L j i}=\sqrt{2} N_{2 j}^{*} C_{L 1 i}+N_{3 j}^{*} C_{L 2 i}, \\
& H_{R j i}=\sqrt{2} N_{2 j} C_{R 1 i}-N_{4 j} C_{R 2 i} .
\end{aligned}
$$

The chargino-quark-squark interactions:

$$
\begin{aligned}
\mathcal{L}= & i \frac{g}{\sqrt{2}} \tilde{d}_{k}^{\dagger} \bar{\omega}_{i}^{c}\left(A_{L i}^{k} \frac{1-\gamma_{5}}{2}+A_{R i}^{k} \frac{1+\gamma_{5}}{2}\right) u+\text { H.c. } \\
& A_{L i}^{k}=\sqrt{2}\left(C_{L 1 i} S_{d 1 k}^{*}-\kappa_{d} C_{L 2 i} S_{d 2 k}^{*}\right) \\
& A_{R i}^{k}=\sqrt{2} \kappa_{u} C_{R 2 i} S_{d 1 k}^{*}, \\
\mathcal{L}= & i \frac{g}{\sqrt{2}} \tilde{u}_{k}^{\dagger} \bar{\omega}_{i}\left(B_{L i}^{k} \frac{1-\gamma_{5}}{2}+B_{R i}^{k} \frac{1+\gamma_{5}}{2}\right) d+\text { H.c, } \\
& B_{L i}^{k}=\sqrt{2}\left(C_{R 1 i}^{*} S_{u 1 k}^{*}-\kappa_{u} C_{R 2 i}^{*} S_{u 2 k}^{*}\right) \\
& B_{R i}^{k}=\sqrt{2} \kappa_{d} C_{L 2 i}^{*} S_{u 1 k}^{*},
\end{aligned}
$$

where ' $u$ ' and 'd' denote up-type and down-type particles, respectively, and $\kappa_{u}$ and $\kappa_{d}$ are defined by

$$
\kappa_{u}=\frac{m_{u}}{\sqrt{2} \sin \beta M_{W}}, \quad \kappa_{d}=\frac{m_{d}}{\sqrt{2} \cos \beta M_{W}} .
$$

The neutralino-quark-squark interactions:

$$
\begin{gathered}
\mathcal{L}=i \frac{g}{\sqrt{2}} \tilde{u}_{k}^{\dagger} \bar{\chi}_{j}\left(F_{L j}^{k} \frac{1-\gamma_{5}}{2}+F_{R j}^{k} \frac{1+\gamma_{5}}{2}\right) u+\text { H.c., } \\
F_{L j}^{k}=\left[\left(2 Q_{u}-1\right) \tan \theta_{W} N_{1 j}+N_{2 j}\right] S_{u 1 k}^{*}+\sqrt{2} \kappa_{u} N_{4 j} S_{u 2 k}^{*}, \\
F_{R j}^{k}=2 Q_{u} \tan \theta_{W} N_{1 j}^{*} S_{u 2 k}^{*}-\sqrt{2} \kappa_{u} N_{4 j}^{*} S_{u 1 k}^{*}, \\
\mathcal{L}=i \frac{g}{\sqrt{2}} \tilde{d}_{k}^{\dagger} \bar{\chi}_{j}\left(G_{L j}^{k} \frac{1-\gamma_{5}}{2}+G_{R j}^{k} \frac{1+\gamma_{5}}{2}\right) d+\text { H.c., } \\
G_{L j}^{k}=\left[\left(2 Q_{d}+1\right) \tan \theta_{W} N_{1 j}-N_{2 j}\right] S_{d 1 k}^{*}+\sqrt{2} \kappa_{d} N_{3 j} S_{d 2 k}^{*}, \\
G_{R j}^{k}=2 Q_{d} \tan \theta_{W} N_{1 j}^{*} S_{d 2 k}^{*}-\sqrt{2} \kappa_{d} N_{3 j}^{*} S_{d 1 k}^{*} .
\end{gathered}
$$

The gluino-quark-squark interactions:

$$
\mathcal{L}=i \sqrt{2} g_{3} \tilde{q}_{k}^{\dagger} T_{3}^{a} \overline{\tilde{g}}^{a}\left(S_{q 1 k}^{*} \frac{1-\gamma_{5}}{2}+S_{q 2 k}^{*} \frac{1+\gamma_{5}}{2}\right) q+\text { H.c. },
$$

where $T_{3}^{a}$ 's represent the generators of the $\mathrm{SU}(3)$ group, and the color indices are understood. 
The squark-squark-W boson interactions:

$$
\begin{gathered}
\mathcal{L}=i \frac{g}{\sqrt{2}} D_{k l}\left(\tilde{u}_{k}^{\dagger} \partial^{\mu} \tilde{d}_{l}-\tilde{d}_{l} \partial^{\mu} \tilde{u}_{k}^{\dagger}\right) W_{\mu}^{\dagger}+\text { H.c. }, \\
D_{k l}=S_{u 1 k}^{*} S_{d 1 l} .
\end{gathered}
$$

The interaction Lagrangians for the lepton and slepton sector are obtained trivially from these equations.

In our scheme, the SSM parameters which determine the interactions at the electroweak scale are $\tan \beta, A, m_{H}, \tilde{m}_{2}, \tilde{M}, m_{3 / 2}$, and $c$. Although these parameters are not all independent of each other, they can have various sets of values depending on assumptions for underlying GUTs and parameter values. For instance, the phase $\theta$ may be related to the phase $\alpha$ by taking specific values for them at the GUT scale [9]. However, if their GUT-scale values are arbitrary, such a relation is not obtained. Therefore, for simplicity, we take those parameters independent and assume only rough constraints coming from theoretical and experimental considerations.

\section{Constraints from EDMs}

The $C P$-violating interactions of the SSM give rise to the EDMs of the neutron and the electron at the one-loop level. The exchanged particles in the loop diagrams are charginos, neutralinos, or gluinos with squarks or sleptons. Among these diagrams, both the neutron and the electron EDMs generally receive dominant contributions from the chargino-loop diagrams, which are approximately proportional to $\sin \theta$. For an unsuppressed value of the $C P$-violating phase $\theta$, the experimental upper bounds on the EDMs impose the constraints that the squarks and sleptons should be heavier than $1 \mathrm{TeV}[8]$.

The constraints on the squark and slepton masses are relaxed, if the EDMs do not receive contributions from the chargino-loop diagrams. These chargino contributions become small as the magnitude of $\theta$ decreases, irrespective of the value of another $C P$-violating phase $\alpha$. For a sufficiently small value of $\theta$, therefore, it is expected that the squarks and sleptons can have relatively small masses even if $\alpha$ is of order unity [10].

Assuming $\theta \ll 1$ and $\alpha \sim 1$, the SSM parameters are constrained by the gluino and neutralino contributions to the neutron EDM, and the neutralino contribution to the electron EDM. The gluino contribution to the EDM of a quark $q$ is obtained 
from the Lagrangian in Eq. (17) as

$$
d_{q}^{G} / e=\frac{2 \alpha_{3}}{3 \pi} Q_{q} \sum_{k=1}^{2} \operatorname{Im}\left(S_{q 1 k}^{*} S_{q 2 k}\right) \frac{\tilde{m}_{3}}{\tilde{M}_{q k}^{2}} I\left(\frac{\tilde{m}_{3}^{2}}{\tilde{M}_{q k}^{2}}, \frac{m_{q}^{2}}{\tilde{M}_{q k}^{2}}\right) .
$$

From the Lagrangians in Eqs. (15) and (16) the neutralino contribution to the EDM of a quark or a lepton $f$ becomes

$$
\begin{aligned}
d_{f}^{N} / e & =\frac{\alpha_{2}}{8 \pi} Q_{f} \sum_{k=1}^{2} \sum_{j=1}^{4} \operatorname{Im}\left(K_{j}^{k}\right) \frac{\tilde{m}_{\chi j}}{\tilde{M}_{f k}^{2}} I\left(\frac{\tilde{m}_{\chi j}^{2}}{\tilde{M}_{f k}^{2}}, \frac{m_{f}^{2}}{\tilde{M}_{f k}^{2}}\right), \\
K_{j}^{k} & =F_{L j}^{k} F_{R j}^{k *} \quad\left(T_{3 f}=\frac{1}{2}\right), \quad G_{L j}^{k} G_{R j}^{k *} \quad\left(T_{3 f}=-\frac{1}{2}\right) .
\end{aligned}
$$

For a quark or a lepton of the first generation, the argument $m_{f}^{2} / \tilde{M}_{f k}^{2}$ can be safely neglected. The function $I(r, s)$ is written for $s=0$ as

$$
I(r, 0)=\frac{1}{2(1-r)^{2}}\left(1+r+\frac{2 r}{1-r} \log r\right) .
$$

In terms of the up-quark and down-quark EDMs, the neutron EDM is given by $d_{n}=\left(4 d_{d}-d_{u}\right) / 3$ from the nonrelativistic quark model.

The gluino contribution to the neutron EDM depends on the squark and gluino masses. For the squark masses given, the predicted magnitude of the EDM decreases as the gluino mass, which is determined by $\tilde{m}_{2}$, increases. The neutralino contributions could also be suppressed by heavy neutralinos, whose masses depend on $\tilde{m}_{2}$, $m_{H}$, and $\tan \beta$. However, the lightest neutralino should be lighter than any squarks or sleptons on cosmological grounds. All the neutralinos cannot be arbitrarily heavy. If $\tilde{m}_{2}$ is large compared to the squark and slepton masses, $\left|m_{H}\right|$ has to be small. In this case, the lightest neutralino has a mass smaller than $\left|m_{H}\right|$ [11]. The lighter chargino mass is around $\left|m_{H}\right|$.

We show in Fig. 2 the absolute value of the EDMs induced by light squarks and sleptons with $\theta=0$ and $\alpha=\pi / 4$ as a function of $\tilde{m}_{2}$. Curves (i) and (ii) respectively represent the EDMs of the neutron and the electron. The other parameters are taken as $\tan \beta=2,\left|m_{H}\right|=100 \mathrm{GeV}$, and $|A| m_{3 / 2}=\tilde{M}=200 \mathrm{GeV}$. The masses of squarks and sleptons, excluding top and bottom squarks, become approximately equal to 200 $\mathrm{GeV}$. The signs of the curves are both positive. In the mass ranges where curves are not drawn, the lighter chargino is lighter than $62 \mathrm{GeV}$, which is ruled out by experiments [12]. The experimental bounds on the neutron and electron EDMs are given by $\left|d_{n}\right|<1 \times 10^{-25} e \mathrm{~cm}$ and $\left|d_{e}\right|<1 \times 10^{-26} e \mathrm{~cm}$, respectively [12]. The predicted value of the neutron EDM, which is dominated by the gluino contribution, lies within 
the experimental bound if $\tilde{m}_{2}>500 \mathrm{GeV}$. The electron EDM is consistent with the experimental bound in all the range of $\tilde{m}_{2}$. The EDMs do not vary much with $\tan \beta$ in the range $\tan \beta \sim 1-10$.

The above numerical analysis shows that, even if squarks, sleptons, one chargino, and some of neutralinos have masses of order $100 \mathrm{GeV}$, the phase $\alpha$ can be of order unity without causing inconsistency for the EDMs. However, gluinos should be heavier than $1 \mathrm{TeV}$. In these parameter ranges the mass-squared matrix of top squarks becomes a source of $C P$ violation. The interactions with the top squarks can thus induce $C P$-violating phenomena at the electroweak scale. On the other hand, the mass-squared matrices of the other squarks and sleptons do not lead to sizable $C P$ violation, owing to the small imaginary parts of their off-diagonal elements.

\section{Decay rate asymmetry}

A nonvanishing value for the decay rate asymmetry $A_{C P}$ in Eq. (1) could be generated if the decay $t \rightarrow \tilde{t} \chi$ is allowed kinematically. The produced top squark and neutralino can become a bottom quark and a $W$ boson by exchanging charginos or bottom squarks as shown in Fig. 1. If $C P$ invariance is violated, the interference of these one-loop diagrams and the tree diagram makes the partial decay rates different between the two decays $t \rightarrow b W^{+}$and $\bar{t} \rightarrow \bar{b} W^{-}$. This difference might also be caused by the decays $t \rightarrow \tilde{t} \tilde{g}$ and $t \rightarrow \tilde{b} \omega$. However, the gluinos should be much heavier than the top quark from the constraint of the neutron EDM. Furthermore, the sum of the experimental lower bounds on gluino and top-squark masses [12] exceeds the top-quark mass. The decay $t \rightarrow \tilde{t} \tilde{g}$ is not allowed kinematically. Since the lighter chargino and the lighter bottom squark are respectively heavier than the lightest neutralino and the lighter top squark, the parameter values which allow the decay $t \rightarrow \tilde{b} \omega$ are more restricted than for the decay $t \rightarrow \tilde{t} \chi$. In fact, it turns out that the former decay is forbidden kinematically in most region of parameter space.

The decay rate asymmetry is obtained as

$$
\begin{aligned}
A_{C P} & =\frac{\alpha_{2}}{2}\left[\left\{m_{t}^{2}+m_{b}^{2}-2 M_{W}^{2}+\frac{\left(m_{t}^{2}-m_{b}^{2}\right)^{2}}{M_{W}^{2}}\right\} \sqrt{\lambda\left(m_{t}^{2}, M_{W}^{2}, m_{b}^{2}\right)}\right]^{-1}\left(T^{a}+T^{b}\right), \\
T^{a} & =\sum_{i=1}^{2} \sum_{j} \sum_{k} \sum_{n=1}^{8} \operatorname{Im}\left(X_{i j k}^{n}\right) I_{n}\left(\tilde{m}_{\omega i}, \tilde{m}_{\chi j}, \tilde{M}_{t k}\right),
\end{aligned}
$$




$$
T^{b}=\sum_{l=1}^{2} \sum_{j} \sum_{k} \sum_{n=1}^{4} \operatorname{Im}\left(Y_{l j k}^{n}\right) J_{n}\left(\tilde{M}_{b l}, \tilde{m}_{\chi j}, \tilde{M}_{t k}\right)
$$

where coefficients $X_{i j k}^{n}, Y_{l j k}^{n}$ and functions $I_{n}\left(\tilde{m}_{\omega i}, \tilde{m}_{\chi j}, \tilde{M}_{t k}\right), J_{n}\left(\tilde{M}_{b l}, \tilde{m}_{\chi j}, \tilde{M}_{t k}\right)$ are given in Appendix. The dummy suffixes $j$ and $k$ run for kinematically allowed pairs of $\tilde{t}_{k}$ and $\chi_{j}$. The kinematic function $\lambda(a, b, c)$ is defined by

$$
\lambda(a, b, c)=a^{2}+b^{2}+c^{2}-2 a b-2 b c-2 c a .
$$

The terms $T^{a}$ and $T^{b}$ respectively come from the contributions of the diagrams (a) and (b) in Fig. 1.

We show in Figs. 3 and the absolute value of the asymmetry $A_{C P}$ as a function of $\tilde{m}_{2}$ for $\theta=0$ and $\alpha=\pi / 4$. The other parameters are taken so as to satisfy the kinematical condition $m_{t}>\tilde{M}_{t 1}+\tilde{m}_{\chi 1}$ as well as the experimental constraints on the squark and chargino masses [12]. The lighter top squark, which is the lightest among the squarks and sleptons, should be heavier than the lightest neutralino. The parameter values are taken in Fig. 3 as $\tan \beta=2,\left|m_{H}\right|=100 \mathrm{GeV}$, and the values of $\tilde{M}$ and $c$ given in Table $\mathbb{1}$, and in Fig. 4 as $\tan \beta=35,\left|m_{H}\right|=80 \mathrm{GeV}$, and the values of $\tilde{M}$ and $c$ given in Table 2. We take $|A| m_{3 / 2}=\tilde{M}$. The top-quark mass is taken to be $180 \mathrm{GeV}$. In Tables 1 and 2, resultant values of the lighter top-squark mass are also given. In the smaller mass ranges where curves are not drawn, the lighter chargino mass is smaller than $62 \mathrm{GeV}$. For large values of $\tilde{m}_{2}$ in Fig. 3, the decay $t \rightarrow \tilde{t} \chi$ is not allowed kinematically.

The asymmetry $A_{C P}$ is of order $10^{-3}$ in a wide region of the parameter space where the decay $t \rightarrow \tilde{t} \chi$ is allowed kinematically. For $\tilde{m}_{2}>500 \mathrm{GeV}$, the predicted value of the neutron EDM does not contradict its experimental bound. The electron EDM is also predicted to be within its experimental bound for all the range of $\tilde{m}_{2}$. This amount of $A_{C P}$ necessitates $t \bar{t}$ pairs of order $10^{6}$ for detection. Since $t \bar{t}$ pairs are expected to be produced at a rate of order $10^{7}$ at LHC, the asymmetry will be detectable in the near future. The signs of all the curves are negative. The spikes of curves (ii) and (iii) in Fig. 1 arise from the opening of the threshold for the decay $t \rightarrow \tilde{t}_{1} \chi_{2}$. For the smaller mass ranges of these curves and all the ranges of the other curves shown, allowed two-body decays of the top quark are only $t \rightarrow b W$ and $t \rightarrow \tilde{t}_{1} \chi_{1}$.

The interactions which induce a nonvanishing value of $A_{C P}$ also yield a rate difference between the two decays $t \rightarrow \tilde{t} \chi$ and $\bar{t} \rightarrow \tilde{t}^{*} \chi$. These decay widths satisfy 
the relation

$$
\Gamma\left(t \rightarrow b W^{+}\right)-\Gamma\left(\bar{t} \rightarrow \bar{b} W^{-}\right)=-\left\{\Gamma(t \rightarrow \tilde{t} \chi)-\Gamma\left(\bar{t} \rightarrow \tilde{t}^{*} \chi\right)\right\} .
$$

Therefore, the total width of the top quark is the same as that of the anti-top quark, as required by $C P T$ invariance.

We briefly comment on some problems encountered when rates of the decays $t \rightarrow b W^{+}$and $\bar{t} \rightarrow \bar{b} W^{-}$are measured in experiments. If these two decays occur at different rates, so do the two decays $t \rightarrow \tilde{t} \chi$ and $\bar{t} \rightarrow \tilde{t}^{*} \chi$. In most of the parameter region where the asymmetry $A_{C P}$ is sizable, the top squark decays sequentially as $\tilde{t} \rightarrow b \omega, \omega \rightarrow W^{*} \chi$, where $W^{*}$ denotes the virtual state of $W$. Consequently, the decay $t \rightarrow \tilde{t} \chi$ results in a final state which contains the same visible particles as the decay $t \rightarrow b W$. Owing to the relation in Eq. (25), a naive asymmetry between specific particles and their antiparticles in the final states of $t \rightarrow b W^{+}$and $\bar{t} \rightarrow \bar{b} W^{-}$ compensates that in the final states of $t \rightarrow \tilde{t} \chi$ and $\bar{t} \rightarrow \tilde{t}^{*} \chi$. In order to measure $A_{C P}$, therefore, some way is needed to distinguish $t \rightarrow b W$ from $t \rightarrow \tilde{t} \chi$. In addition, if the top squark has such a small mass, pairs of $\tilde{t} \tilde{t}^{*}$ are directly produced at a rate larger than the pair production of $t \bar{t}$. Since the decays $t \rightarrow b W$ and $\tilde{t} \rightarrow b \omega$ yield the same visible particles, we also need a way to distinguish between them. These distinctions should be made by detailed analyses of energy spectra of the particles in the final state.

The non-standard decay $t \rightarrow \tilde{t} \chi$, required by a nonvanishing value of the asymmetry $A_{C P}$, may be detected at Tevatron, if it has a large branching ratio. In Figs. 5 and 6 the branching ratio of $t \rightarrow \tilde{t}_{1} \chi_{1}$ is shown for the same parameter values as in Figs. 3 and 4 . The branching ratio becomes around 0.2. As discussed above, the final states of the decays $t \rightarrow \tilde{t} \chi, t \rightarrow b W$, and $\tilde{t} \rightarrow b \omega$ arising from a $\tilde{t}^{*}$ pair production have the same visible particles. Energy spectra of these particles have to be examined in detail to find the non-standard decay mode. In fact, it is not ruled out 13 that this decay may have escaped detection, even if its branching ratio is comparable to that of the standard decay $t \rightarrow b W$.

It may happen by some reasons that the squarks of the third generation are of order $100 \mathrm{GeV}$ while the other squarks and sleptons are heavier than $1 \mathrm{TeV}$. Then, the EDMs of the neutron and the electron do not impose constraints on the $C P$ violating phases $\theta$ and $\alpha$ or the mass parameters $\tilde{m}_{2}$ and $\left|m_{H}\right|$. We have computed the $C P$ asymmetry $A_{C P}$ without imposing the constraints of the EDMs, searching for a parameter region which gives a large value for $A_{C P}$. However, taking into account experimental lower bounds on the gluino and top-squark masses, the decay 
$t \rightarrow \tilde{t} \tilde{g}$, which might lead to a large asymmetry [4], is not allowed kinematically. The decay $t \rightarrow \tilde{b} \omega$ is also not allowed in most region of parameter space. The asymmetry $A_{C P}$ induced by the decay $t \rightarrow \tilde{t} \chi$ is at most around $3 \times 10^{-3}$, and not significantly large compared to the asymmetry obtained under the constraints of the EDMs. This numerical result agrees with Ref. [6] but is much smaller than the result of Ref. [5].

\section{Conclusions}

We have considered a possibility that $C P$ violation is observed at the electroweak energy scale within the framework of the SSM. In this model, the EDMs of the neutron and the electron severely constrain $C P$-violating phases and masses of $R$ odd particles. However, we showed that there is a parameter region where squarks, sleptons, one chargino, and some of neutralinos have masses of order $100 \mathrm{GeV}$ and one $C P$-violating phase $\alpha$ is of order unity. In this region, the mass-squared matrix of top squarks becomes a new source of $C P$ violation. This source can affect the production and decay of the top quark, which is and will be studied extensively in experiments.

Among phenomena possibly induced by the new source of $C P$ violation, we studied a decay rate asymmetry of the top quark. If the two-body decay $t \rightarrow \tilde{t} \chi$ is allowed, the decay widths for $t \rightarrow b W^{+}$and $\bar{t} \rightarrow \bar{b} W^{-}$could be different from each other. It was shown that this asymmetry is of order $10^{-3}$ in a wide region of the parameter space where the decay $t \rightarrow \tilde{t} \chi$ occurs. Since $10^{7}$ pairs of top quarks are expected to be produced at LHC, such an amount of asymmetry will be detectable. The decay rate asymmetry necessitates the two-body decay mode of the top quark different from the standard decay $t \rightarrow b W$, so that the parameter region may be probed at Tevatron. If further experimental analyses find the non-standard decay of the top quark, the decay rate asymmetry will be worth measuring at LHC.

The new source of $C P$ violation could affect the angular distributions of the particles produced by the top-quark decay. Although the decay rate asymmetry becomes nonvanishing only in a parameter region restricted by the mass relation $m_{t}>\tilde{M}_{t 1}+\tilde{m}_{\chi 1}$, the $C P$ asymmetries of the angular distributions can occur in wider parameter region. It would be interesting to study these asymmetries taking into consideration the results of this paper. A light top squark and an unsuppressed $C P$-violating phase could also affect the $B$-meson system.

The baryon asymmetry of our universe may have been generated at the electroweak phase transition. This scenario could be realized in the SSM, if there exists 
a light top squark with $C P$-violating interactions [10]. This possibility will become more plausible, if $C P$ violation in the top-quark system is found.

\section{Acknowledgments}

We thank J. Arafune, J. Kamoshita, A. Sugamoto, I. Watanabe, and P. Zerwas for discussions. The work of M.A. is supported in part by the Grant-in-Aid for Scientific Research from the Ministry of Education, Science and Culture, Japan. This work is supported in part by the Grant-in-Aid for Scientific Research (No. 08044089) from the Ministry of Education, Science and Culture, Japan.

\section{Appendix}

The coefficients $X_{i j k}^{n}$ and the functions $I_{n}\left(\tilde{m}_{\omega}, \tilde{m}_{\chi}, \tilde{M}_{t}\right)$ in Eq. (23) are defined by

$$
\begin{aligned}
& X_{i j k}^{1}=B_{L i}^{k *} F_{L j}^{k} H_{L j i}^{*}, \quad X_{i j k}^{2}=B_{L i}^{k *} F_{L j}^{k} H_{R j i}^{*}, \quad X_{i j k}^{3}=B_{L i}^{k *} F_{R j}^{k} H_{L j i}^{*}, \\
& X_{i j k}^{4}=B_{L i}^{k *} F_{R j}^{k} H_{R j i}^{*}, \quad X_{i j k}^{5}=B_{R i}^{k *} F_{L j}^{k} H_{L j i}^{*}, \quad X_{i j k}^{6}=B_{R i}^{k *} F_{L j}^{k} H_{R j i}^{*}, \\
& X_{i j k}^{7}=B_{R i}^{k *} F_{R j}^{k} H_{L j i}^{*}, \quad X_{i j k}^{8}=B_{R i}^{k *} F_{R j}^{k} H_{R j i}^{*}, \\
& I_{1}=-\frac{1}{2} \tilde{m}_{\omega} \tilde{m}_{\chi}\left\{m_{t}^{2}+m_{b}^{2}-2 M_{W}^{2}+\frac{1}{M_{W}^{2}}\left(m_{t}^{2}-m_{b}^{2}\right)^{2}\right\} \log \left|\frac{A+B}{A-B}\right|, \\
& I_{2}=-\left\{m_{t}^{2}\left(m_{t}^{2}-m_{b}^{2}+3 M_{W}^{2}-\tilde{M}_{t}^{2}+2 \tilde{m}_{\omega}^{2}-\tilde{m}_{\chi}^{2}\right)+\left(m_{b}^{2}-3 M_{W}^{2}\right)\left(\tilde{M}_{t}^{2}-\tilde{m}_{\chi}^{2}\right)\right\} B \\
& +\frac{1}{2}\left(m_{t}^{2}\left(2 m_{b}^{2}-2 \tilde{M}_{t}^{2}+\tilde{m}_{\omega}^{2}\right)-m_{b}^{2}\left(2 \tilde{M}_{t}^{2}-\tilde{m}_{\chi}^{2}\right)+2\left(\tilde{M}_{t}^{2}-\tilde{m}_{\omega}^{2}\right)\left(\tilde{M}_{t}^{2}-\tilde{m}_{\chi}^{2}\right)\right. \\
& +\frac{1}{M_{W}^{2}}\left[m_{t}^{2}\left\{m_{t}^{2} \tilde{m}_{\omega}^{2}-m_{b}^{2}\left(\tilde{m}_{\omega}^{2}+\tilde{m}_{\chi}^{2}\right)-\left(\tilde{M}_{t}^{2}-\tilde{m}_{\omega}^{2}\right)\left(\tilde{m}_{\omega}^{2}-\tilde{m}_{\chi}^{2}\right)\right\}\right. \\
& \left.\left.+m_{b}^{2}\left\{m_{b}^{2} \tilde{m}_{\chi}^{2}+\left(\tilde{M}_{t}^{2}-\tilde{m}_{\chi}^{2}\right)\left(\tilde{m}_{\omega}^{2}-\tilde{m}_{\chi}^{2}\right)\right\}\right]\right) \log \left|\frac{A+B}{A-B}\right| \\
& I_{3}=-m_{t} \tilde{m}_{\omega}\left[2\left(m_{t}^{2}-m_{b}^{2}-2 M_{W}^{2}\right) B+\frac{1}{2}\left\{2 m_{t}^{2}-m_{b}^{2}-2 M_{W}^{2}-\tilde{M}_{t}^{2}+2 \tilde{m}_{\omega}^{2}-\tilde{m}_{\chi}^{2}\right.\right. \\
& \left.\left.-\frac{1}{M_{W}^{2}}\left(m_{t}^{2}-m_{b}^{2}\right)\left(\tilde{m}_{\omega}^{2}-\tilde{m}_{\chi}^{2}\right)\right\} \log \left|\frac{A+B}{A-B}\right|\right] \\
& I_{4}=-3 m_{t} \tilde{m}_{\chi}\left\{2 M_{W}^{2} B-\frac{1}{2}\left(m_{b}^{2}-\tilde{M}_{t}^{2}+\tilde{m}_{\omega}^{2}\right) \log \left|\frac{A+B}{A-B}\right|\right\} \\
& I_{5}=-m_{b} \tilde{m}_{\chi}\left[2\left(m_{t}^{2}-m_{b}^{2}+M_{W}^{2}\right) B-\frac{1}{2}\left\{m_{t}^{2}-2 m_{b}^{2}+2 M_{W}^{2}+\tilde{M}_{t}^{2}+\tilde{m}_{\omega}^{2}-2 \tilde{m}_{\chi}^{2}\right.\right.
\end{aligned}
$$




$$
\begin{aligned}
& \left.\left.+\frac{1}{M_{W}^{2}}\left(m_{t}^{2}-m_{b}^{2}\right)\left(\tilde{m}_{\omega}^{2}-\tilde{m}_{\chi}^{2}\right)\right\} \log \left|\frac{A+B}{A-B}\right|\right], \\
I_{6}= & \frac{3}{2} m_{b} \tilde{m}_{\omega}\left(m_{t}^{2}-\tilde{M}_{t}^{2}+\tilde{m}_{\chi}^{2}\right) \log \left|\frac{A+B}{A-B}\right|, \\
I_{7}= & -m_{t} m_{b}\left[\left\{m_{t}^{2}-m_{b}^{2}-3 M_{W}^{2}-\tilde{M}_{t}^{2}-2 \tilde{m}_{\omega}^{2}+3 \tilde{m}_{\chi}^{2}\right.\right. \\
& \left.+\frac{1}{m_{t}^{2}}\left(m_{b}^{2}-M_{W}^{2}\right)\left(\tilde{M}_{t}^{2}-\tilde{m}_{\chi}^{2}\right)\right\} B \\
& \left.-\frac{1}{2}\left\{2 M_{W}^{2}-\tilde{m}_{\omega}^{2}-\tilde{m}_{\chi}^{2}-\frac{1}{M_{W}^{2}}\left(\tilde{m}_{\omega}^{2}-\tilde{m}_{\chi}^{2}\right)^{2}\right\} \log \left|\frac{A+B}{A-B}\right|\right], \\
I_{8}= & 3 m_{t} m_{b} \tilde{m}_{\omega} \tilde{m}_{\chi} \log \left|\frac{A+B}{A-B}\right|,
\end{aligned}
$$

where

$$
\begin{aligned}
A & =\frac{\left(m_{t}^{2}+M_{W}^{2}-m_{b}^{2}\right)\left(m_{t}^{2}-\tilde{M}_{t}^{2}+\tilde{m}_{\chi}^{2}\right)}{4 M_{W}^{2} m_{t}^{2}}-\frac{M_{W}^{2}+\tilde{m}_{\chi}^{2}-\tilde{m}_{\omega}^{2}}{2 M_{W}^{2}} \\
B & =\frac{1}{4 m_{t}^{2} M_{W}^{2}} \sqrt{\lambda\left(m_{t}^{2}, M_{W}^{2}, m_{b}^{2}\right) \lambda\left(m_{t}^{2}, \tilde{M}_{t}^{2}, \tilde{m}_{\chi}^{2}\right)}
\end{aligned}
$$

The coefficients $Y_{l j k}^{n}$ and the functions $J_{n}\left(\tilde{M}_{b}, \tilde{m}_{\chi}, \tilde{M}_{t}\right)$ are defined by

$$
\begin{gathered}
Y_{l j k}^{1}=D_{k l}^{*} F_{L j}^{k} G_{L j}^{l *}, \quad Y_{l j k}^{2}=D_{k l}^{*} F_{L j}^{k} G_{R j}^{l *}, \quad Y_{l j k}^{3}=D_{k l}^{*} F_{R j}^{k} G_{L j}^{l *}, \\
Y_{l j k}^{4}=D_{k l}^{*} F_{R j}^{k} G_{R j}^{l *}, \\
J_{1}=-\left\{m_{t}^{2}\left(m_{t}^{2}-m_{b}^{2}+M_{W}^{2}+\tilde{M}_{t}^{2}-2 \tilde{M}_{b}^{2}+\tilde{m}_{\chi}^{2}\right)+\left(m_{b}^{2}-3 M_{W}^{2}\right)\left(\tilde{M}_{t}^{2}-\tilde{m}_{\chi}^{2}\right)\right\} B \\
+\frac{1}{2}\left[m_{t}^{2}\left(\tilde{M}_{b}^{2}+\tilde{m}_{\chi}^{2}\right)+m_{b}^{2}\left(\tilde{M}_{t}^{2}+\tilde{m}_{\chi}^{2}\right)-2\left(\tilde{M}_{t}^{2}-\tilde{m}_{\chi}^{2}\right)\left(\tilde{M}_{b}^{2}-\tilde{m}_{\chi}^{2}\right)-2 M_{W}^{2} \tilde{m}_{\chi}^{2}\right. \\
\left.+\frac{1}{M_{W}^{2}}\left\{m_{t}^{2}\left(\tilde{M}_{b}^{2}-\tilde{m}_{\chi}^{2}\right)-m_{b}^{2}\left(\tilde{M}_{t}^{2}-\tilde{m}_{\chi}^{2}\right)\right\}\left(\tilde{M}_{t}^{2}-\tilde{M}_{b}^{2}\right)\right] \log \left|\frac{A^{\prime}+B}{A^{\prime}-B}\right|, \\
J_{2}=-m_{b} \tilde{m}_{\chi}\left[2\left(m_{t}^{2}-m_{b}^{2}+M_{W}^{2}\right) B-\frac{1}{2}\left\{m_{t}^{2}+m_{b}^{2}-M_{W}^{2}+\tilde{M}_{t}^{2}+\tilde{M}_{b}^{2}-2 \tilde{m}_{\chi}^{2}\right.\right. \\
\left.\left.\quad-\frac{1}{M_{W}^{2}}\left(m_{t}^{2}-m_{b}^{2}\right)\left(\tilde{M}_{t}^{2}-\tilde{M}_{b}^{2}\right)\right\} \log \left|\frac{A^{\prime}+B}{A^{\prime}-B}\right|\right], \\
J_{3}=\frac{m_{t}}{m_{b}} J_{2}, \\
J_{4}=-m_{t} m_{b}\left[\left\{m_{t}^{2}-m_{b}^{2}-M_{W}^{2}-3 \tilde{M}_{t}^{2}+2 \tilde{M}_{b}^{2}+\tilde{m}_{\chi}^{2}\right.\right. \\
\left.+\frac{1}{m_{t}^{2}}\left(m_{b}^{2}-M_{W}^{2}\right)\left(\tilde{M}_{t}^{2}-\tilde{m}_{\chi}^{2}\right)\right\} B-\frac{1}{2}\left\{m_{t}^{2}+m_{b}^{2}-\tilde{M}_{t}^{2}-\tilde{M}_{b}^{2}-2 \tilde{m}_{\chi}^{2}\right.
\end{gathered}
$$




$$
\left.\left.-\frac{1}{M_{W}^{2}}\left(m_{t}^{2}-m_{b}^{2}-\tilde{M}_{t}^{2}+\tilde{M}_{b}^{2}\right)\left(\tilde{M}_{t}^{2}-\tilde{M}_{b}^{2}\right)\right\} \log \left|\frac{A^{\prime}+B}{A^{\prime}-B}\right|\right],
$$

where

$$
A^{\prime}=\frac{\left(m_{t}^{2}+M_{W}^{2}-m_{b}^{2}\right)\left(m_{t}^{2}+\tilde{M}_{t}^{2}-\tilde{m}_{\chi}^{2}\right)}{4 M_{W}^{2} m_{t}^{2}}-\frac{M_{W}^{2}+\tilde{M}_{t}^{2}-\tilde{M}_{b}^{2}}{2 M_{W}^{2}} .
$$




\section{References}

[1] For general reviews, see e.g. J.F. Donoghue, B.R. Holstein, and G. Valencia, Int. J. Mod. Phys. A2 (1987) 319;

W. Grimus, Fortschr. Phys. 36 (1988) 201.

[2] G. Eilam, J.L. Hewett, and A. Soni, Phys. Rev. Lett. 67 (1991) 1979;

D. Atwood and A. Soni, Phys. Rev. D45 (1992) 2405;

C. R. Schmidt and M. E. Peskin, Phys. Rev. Lett. 69 (1992) 410;

B. Grzadkowski and J.F. Gunion, Phys. Lett. B287 (1992) 237;

D. Atwood, G. Eilam, A. Soni, R.R. Mendel, and R. Migneron, Phys. Rev. Lett. 70 (1993) 1364;

D. Chang, W.-Y. Keung, and I. Phillips, Nucl. Phys. B408 (1993) 286;

W. Bernreuther and A. Brandenburg, Phys. Lett. B314 (1993) 104;

F. Cuypers and S. D. Rindani, Phys. Lett. B343 (1995) 333;

P. Poulose and S.D. Rindani, Phys. Lett. B349 (1995) 379;

B. Grzadkowski and Z. Hioki, Phys. Lett. B391 (1997) 172;

T. Hasuike, T. Hattori, T. Hayashi, and S. Wakaizumi, Z. Phys. C76 (1997) 127.

[3] C. R. Schmidt, Phys. Lett. B 293 (1992) 111;

E. Christova and M. Fabbrichesi, Phys. Lett. B315 (1993) 338;

B. Grzadkowski and W.-Y. Keung, Phys. Lett. B316 (1993) 137;

A. Bartl, E. Christova, and W. Majerotto, Nucl. Phys. B460 (1996) 235;

A. Bartl, E. Christova, T. Gajdosik, and W. Majerotto, Nucl. Phys. B507 (1997) 35 .

[4] B. Grzadkowski and W.-Y. Keung, Phys. Lett. B319 (1993) 526.

[5] E. Christova and M. Fabbrichesi, Phys. Lett. B320 (1994) 299.

[6] S. Bar-Shalom, D. Atwood, and A. Soni, UCRHEP-T201.

[7] For reviews, see e.g. H.P. Nilles, Phys. Rep. 110 (1984) 1;

P. Nath, R. Arnowitt, and A.H. Chamseddine, Applied N=1 Supergravity (World Scientific, Singapore, 1984);

H.E. Haber and G.L. Kane, Phys. Rep. 117 (1985) 75.

[8] Y. Kizukuri and N. Oshimo, Phys. Rev. D45 (1992) 1806; D46 (1992) 3025. 
[9] R. Garisto and J.D. Wells, Phys. Rev. D55 (1997) 1611.

[10] M. Aoki, A. Sugamoto, and N. Oshimo, Prog. Theor. Phys. 98 (1997) 1325.

[11] A. Bartl, H. Fraas, W. Majerotto, and N. Oshimo, Phys. Rev. D40 (1989) 1594.

[12] Particle Data Group, Phys. Rev. D54 (1996) 1 (updated, 1997).

[13] J. Sender, Phys. Rev. D54 (1996) 3271. 
Table 1: The values of $\tilde{M}$ and $c$ for curves (i)-(iii) in Fig. 3. The lighter top-squark mass is also given.

\begin{tabular}{cccc} 
& $\tilde{M}(\mathrm{GeV})$ & $\mathrm{c}$ & $\tilde{M}_{t 1}(\mathrm{GeV})$ \\
\hline (i) & 180 & 0.3 & 97 \\
(ii) & 200 & 0.4 & 92 \\
(iii) & 220 & 0.5 & 91
\end{tabular}

Table 2: The values of $\tilde{M}$ and $c$ for curves (i)-(iii) in Fig. 4 . The lighter top-squark mass is also given.

\begin{tabular}{cccc} 
& $\tilde{M}(\mathrm{GeV})$ & $\mathrm{c}$ & $\tilde{M}_{t 1}(\mathrm{GeV})$ \\
\hline (i) & 180 & 0.4 & 101 \\
(ii) & 200 & 0.5 & 95 \\
(iii) & 220 & 0.6 & 93
\end{tabular}

Figure 1: The one-loop diagrams for the top-quark decay producing a bottom quark and a $W$ boson, where $\tilde{t}, \tilde{b}, \omega$, and $\chi$ denote respectively the top squark, bottom squark, chargino, and neutralino.

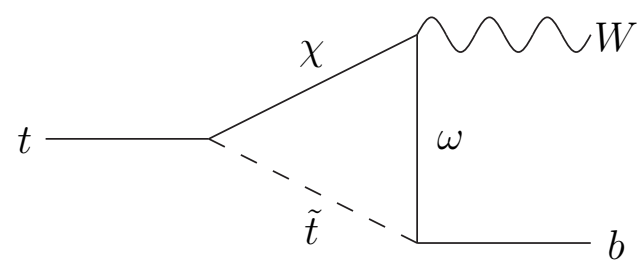

(a)

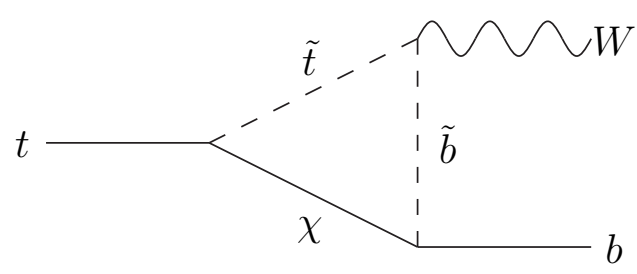

(b) 
Figure 2: The EDMs of the neutron and the electron, being respectively represented by curves (i) and (ii), as a function of $\tilde{m}_{2}$ for $\alpha=\pi / 4$ and $\theta=0$. The other parameters are taken as $\tan \beta=2,\left|m_{H}\right|=100 \mathrm{GeV}$, and $|A| m_{3 / 2}=\tilde{M}=200 \mathrm{GeV}$.

\section{Electric Dipole Moment (e.cm)}

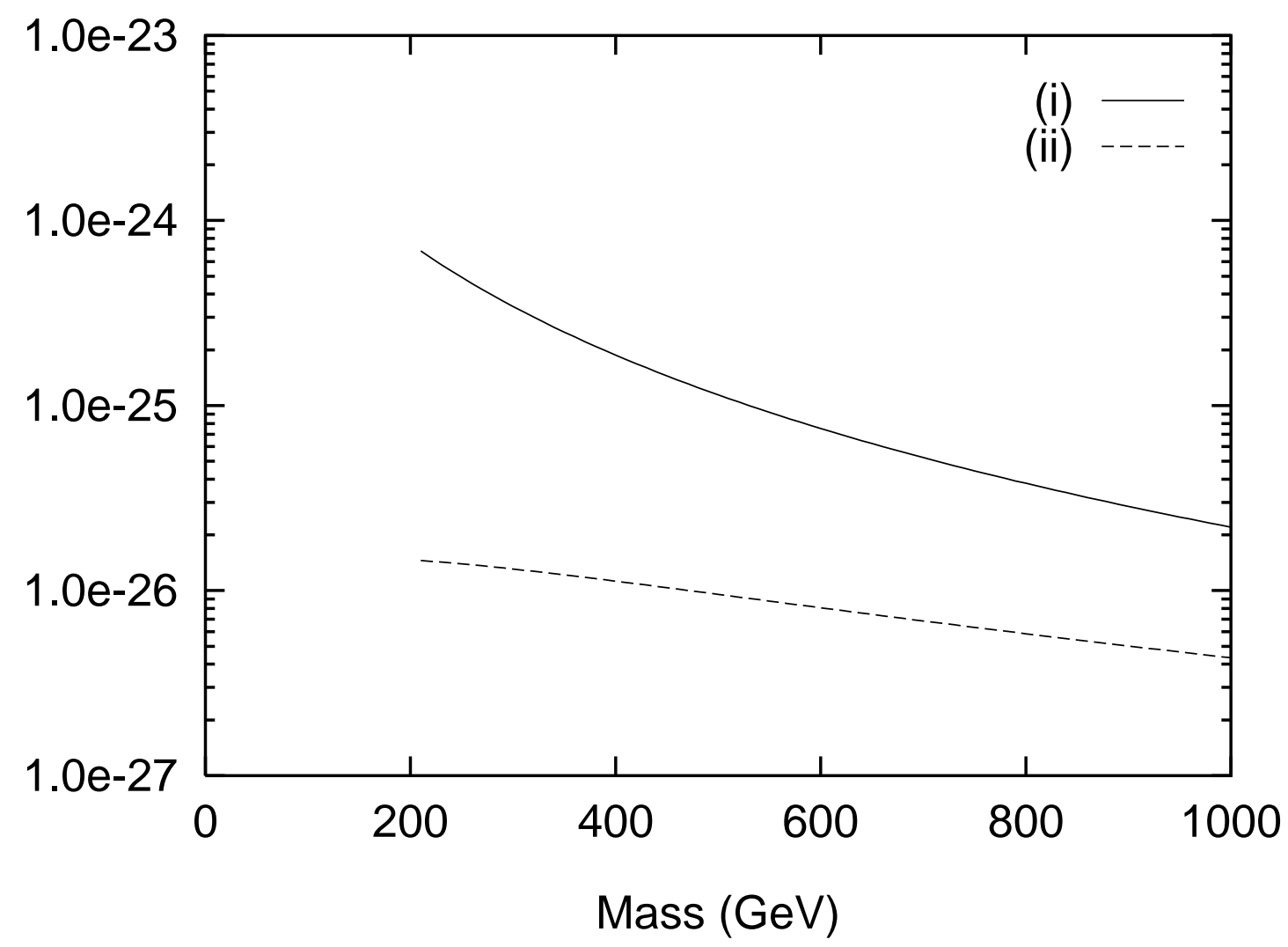


Figure 3: The decay rate asymmetry as a function of $\tilde{m}_{2}$ for $\alpha=\pi / 4$ and $\theta=0$. Three curves correspond to three sets of values for $\tilde{M}$ and $c$ in Table 11. The other parameters are taken as $\tan \beta=2,\left|m_{H}\right|=100 \mathrm{GeV}$, and $|A| m_{3 / 2}=\vec{M}$.

\section{CP Asymmetry}

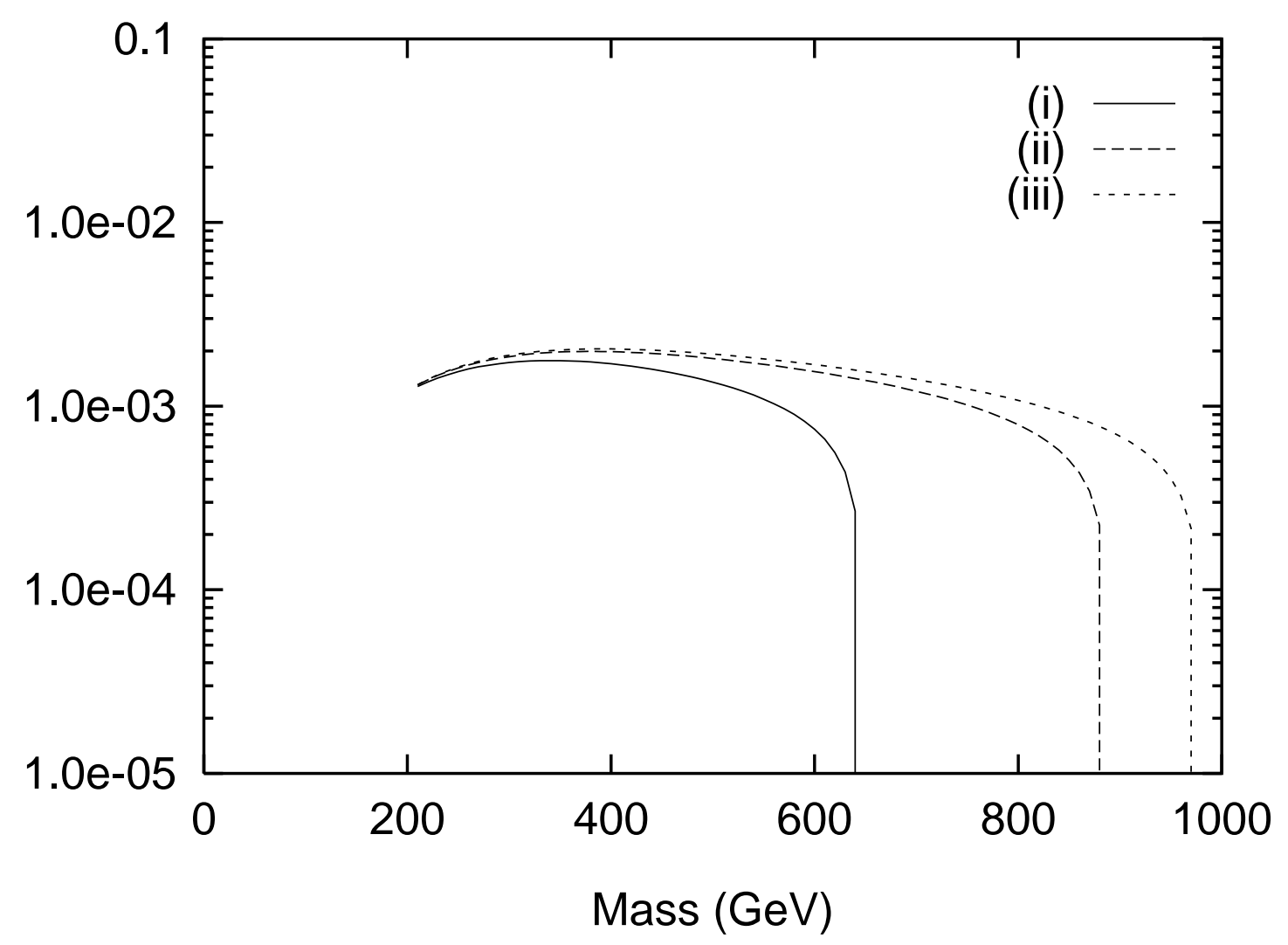


Figure 4: The decay rate asymmetry as a function of $\tilde{m}_{2}$ for $\alpha=\pi / 4$ and $\theta=0$. Three curves correspond to three sets of values for $\tilde{M}$ and $c$ in Table 2 . The other parameters are taken as $\tan \beta=35,\left|m_{H}\right|=80 \mathrm{GeV}$, and $|A| m_{3 / 2}=\dot{M}$.

\section{CP Asymmetry}

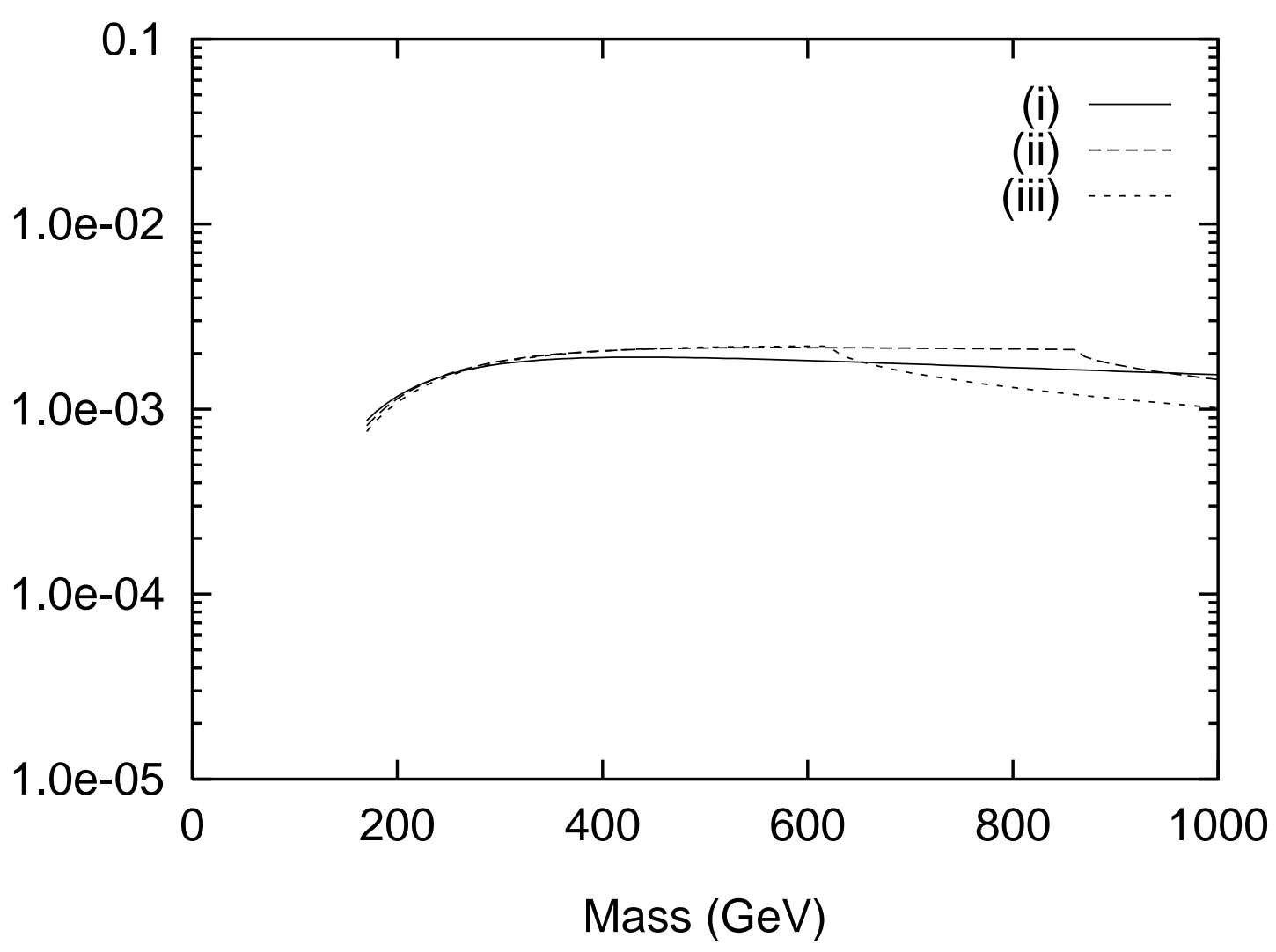


Figure 5: The branching ratio of $t \rightarrow \tilde{t}_{1} \chi_{1}$ as a function of $\tilde{m}_{2}$ for the parameter values in Fig. 3 .

\section{Branching Ratio}

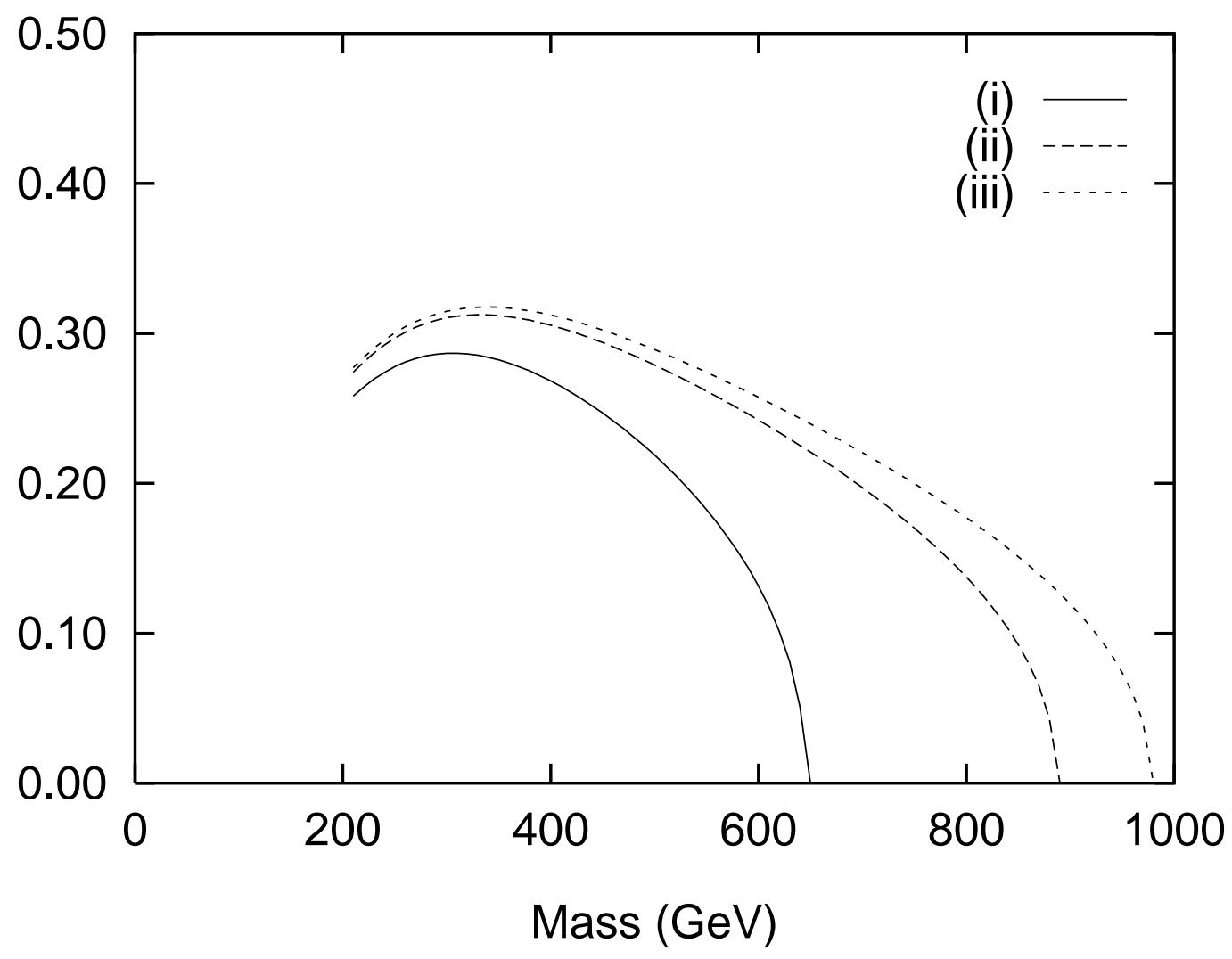


Figure 6: The branching ratio of $t \rightarrow \tilde{t}_{1} \chi_{1}$ as a function of $\tilde{m}_{2}$ for the parameter values in Fig. 4 .

\section{Branching Ratio}

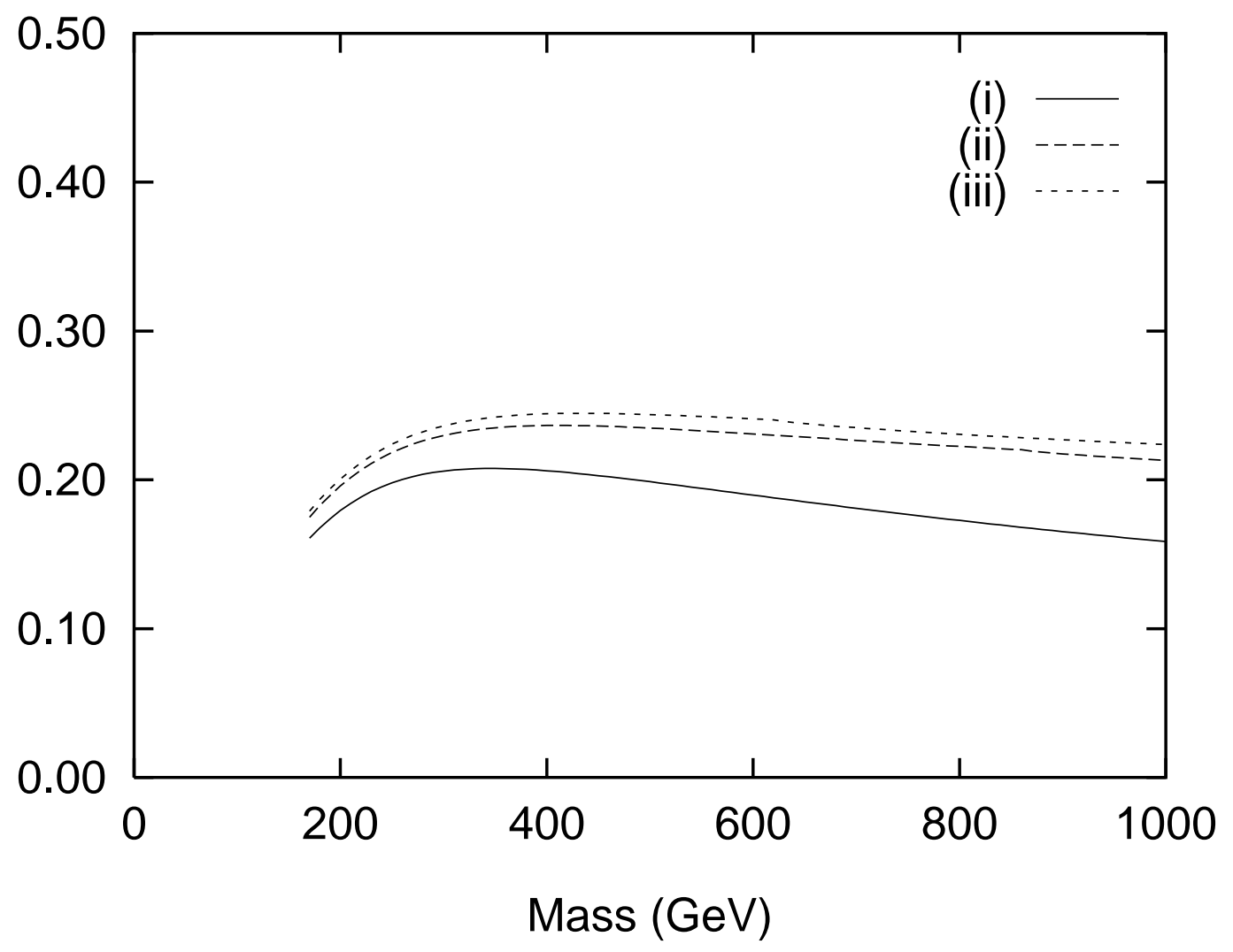

